\title{
Otimização de produção de biomassa para extração lipídica utilizando estirpes da
}

\author{
microalga Scenedesmus sp.
}

Optimization of biomass production for lipid extraction using strains Scenedesmus sp. microalgae

Optimización de la producción de biomasa para la extracción de lípidos utilizando cepas de la

microalga Scenedesmus sp.

Recebido: 07/12/2021 | Revisado: 12/12/2021 | Aceito: 18/12/2021 | Publicado: 02/01/2022

Flávia Costa Oliveira

ORCID: https://orcid.org/0000-0002-5599-6242

Universidade Federal de Uberlândia, Brasil

E-mail: flaviacosta.iub@hotmail.com

Witter Duarte Guerra

ORCID: https://orcid.org/0000-0002-9155-8047 Universidade Federal de Uberlândia, Brasil E-mail: witter_guerra@yahoo.com.br

Jaqueline Elise Garcia Chiesa

ORCID: https://orcid.org/0000-0002-3767-0900

Universidade Federal de Uberlândia, Brasil

E-mail: jaquelineelisegarcia@hotmail.com

Isabela de Souza Dias

ORCID: https://orcid.org/0000-0003-2574-0833

Universidade Federal de Uberlândia, Brasil

E-mail: isabeladias97@gmail.com

Andressa Tironi Vieira

ORCID: https://orcid.org/0000-0003-3662-1346

Universidade Federal de Uberlândia, Brasil

E-mail: dessaqtironi@yahoo.com.br

Wesley da Silva Borges

ORCID: https://orcid.org/0000-0002-9606-0758

Instituto Luterano de Ensino Superior, Brasil

E-mail: wesley.itb@gmail.com

Anizio Marcio de Faria

ORCID: https://orcid.org/0000-0001-6915-8963

Universidade Federal de Uberlândia, Brasil

E-mail: anizio@ufu.br

Antônio Carlos Ferreira Batista

ORCID: https://orcid.org/0000-0001-6313-4565

Universidade Federal de Uberlândia, Brasil

E-mail: batistaacf@ufu.br

\begin{abstract}
Resumo
Espécies de microalgas como a Scenedesmus sp. têm alto potencial de acumulação lipídica em suas células, viabilizando estudos voltados a exploração desses organismos como matéria prima para extração de bio-óleo destinado a produção de biodiesel, potencializando os sistemas de cultivo e colheita de biomassa. Este estudo foi desenvolvido com o objetivo de avaliar técnicas de cultivo da microalga Scenedesmus sp. que possibilite uma maior produção de bio-óleo. O cultivo ocorreu em reatores planos horizontais em acrílico, mantidos em temperatura ambiente a $25^{\circ} \mathrm{C} \pm 1{ }^{\circ} \mathrm{C}$, com controlador de fotoperíodo em 12 horas de luz e 12 horas de escuro, utilizando três lâmpadas fluorescentes brancas de 40 Volts. O meio de cultivo, tempo e a porcentagem de inóculo foram as variáveis otimizadas para o crescimento das algas e rendimento lipídico. Visando minimizar o número de experimentos realizados e o tempo gasto utilizou-se o planejamento fatorial 23 e o planejamento composto central. As análises indicaram que o cultivo de microalga Scenedesmus sp., apresentou melhores rendimentos no teor lipídico, quando submetidos ao cultivo em meio Chu com $20 \%$ de inóculo inicial. No entanto, o acúmulo de lipídeos atinge um valor máximo quando a concentração inicial de meio Chu é de aproximadamente 39,5\%. Verificou-se ainda que a quantidade de dias (tempo) indicou a tendência de maior acúmulo de lipídeos em 20 dias de cultivo. Para essas condições de concentração inicial de meio Chu (39,5\%) e tempo de cultivo (20 dias), o modelo prevê um acúmulo de teor lipídico de aproximadamente 9,74\%.
\end{abstract}

Palavras-chave: Biodiesel; Microalgas; Scenedesmus; Lipídeos; Otimização estatística. 


\begin{abstract}
Microalgae species such as Scenedesmus sp. have a high potential for lipid accumulation in their cells, enabling studies aimed at the exploration of these organisms as raw materials for the bio-oil extraction for biodiesel production, enhancing the biomass cultivation and harvesting systems. This study was developed to evaluate cultivation techniques of the Scenedesmus sp. microalgae that allows greater production of bio-oil. Cultivation took place in horizontal flat acrylic reactors, maintained at room temperature at $25{ }^{\circ} \mathrm{C} \pm 1{ }^{\circ} \mathrm{C}$, with a photoperiod controller in 12 hours of light and 12 hours of darkness, using three 40-volt white fluorescent lamps. The culture medium, time, and percentage of inoculum were the variables optimized for algae growth and lipid yield. To minimize the number of experiments and time, 23 factorial and central composite designs were used. The analyzes indicated that the cultivation of Scenedesmus sp. microalgae presented better yields in the lipid content when submitted to the cultivation in Chu medium with $20 \%$ of the initial inoculum. However, the lipid accumulation reaches a maximum when the initial concentration of Chu medium is approximately 39.5\%. It was also found that the number of days (time) indicated the tendency of greater lipid accumulation in 20 days of cultivation. For these conditions of initial concentration of Chu medium (39.5\%) and cultivation time (20 days), the model predicts an accumulation of lipid content of approximately $9.74 \%$.
\end{abstract}

Keywords: Biodiesel; Microalgae; Scenedesmus; Lipids; Statistical optimization.

\title{
Resumen
}

Especies de microalgas como Scenedesmus sp. tienen un alto potencial de acumulación de lípidos en sus células, lo que permite realizar estudios dirigidos a explorar estos organismos como materia prima para la extracción de bioaceites para la producción de biodiesel, potenciando los sistemas de cultivo y cosecha de biomasa. Este estudio se desarrolló con el objetivo de evaluar técnicas para el cultivo de la microalga Scenedesmus sp. que permite una mayor producción de bioaceite. El cultivo se realizó en reactores acrílicos planos horizontales, mantenidos a temperatura ambiente a $25{ }^{\circ} \mathrm{C} \pm 1{ }^{\circ} \mathrm{C}$, con controlador de fotoperiodo a 12 horas de luz y 12 horas de oscuridad, utilizando tres lámparas fluorescentes blancas de 40 Voltios. El medio de cultivo, el tiempo y el porcentaje de inóculo fueron las variables optimizadas para el crecimiento de algas y el rendimiento de lípidos. Con el objetivo de minimizar el número de experimentos realizados y el tiempo empleado, se utilizaron planificación factorial $2^{3}$ y planificación central compuesta. Los análisis indicaron que el cultivo de microalgas Scenedesmus sp., Mostró mejores rendimientos en contenido de lípidos, cuando se sometió a cultivo en medio Chu con $20 \%$ de inóculo inicial. Sin embargo, la acumulación de lípidos alcanza un valor máximo cuando la concentración inicial de medio Chu es aproximadamente del 39,5\%. También se encontró que el número de días (tiempo) indicó una tendencia hacia una mayor acumulación de lípidos en 20 días de cultivo. Para estas condiciones de concentración inicial de medio Chu (39,5\%) y tiempo de cultivo (20 días), el modelo predice una acumulación de contenido lipídico de aproximadamente 9,74\%.

Palabras clave: Biodiesel; Microalgas; Scenedesmus; Lípidos; Optimización estadística.

\section{Introdução}

Uma opção significativa para a produção de biodiesel, é a exploração de outros organismos fotossintéticos, em que se destacam as microalgas como alternativa verde e renovável, têm-se estabelecido como matéria prima promissora, capazes de acumular alto teor lipídico, gerando uma nova extensão nas indústrias de energias (Shi; Handler; Shonnard, 2019). Protistas, as microalgas constituem um grupo bem heterogêneo de espécies fotossintéticas, capazes de produzir proteínas, hidrocarbonetos, polissacarídeos e lipídeos empregados como fonte de energia potencial aos biocombustíveis (Svenning et al., 2019). Segundo Ramos (2017) o meio de cultivo ideal para produção de energia via microalgas, refere-se a um processo cuidadosamente direcionado a cada espécie em questão, para que os resultados de adaptação e retorno de nutrientes sejam satisfatórios, apresentando um perfil socioeconômico rentável.

Contudo é importante analisar a viabilidade de produção do biodiesel perante o rendimento de óleo por espécies. No que concerne às microalgas, podem chegar a um potencial lipídico de até $70 \%$. Nesta perspectiva, Cavalcante Filho; Buaianin; Benatti (2019) relata em seus estudos que a quantidade de óleo presentes nas cadeias produtivas agrícolas, variam cerca de 47 $\%$ no óleo de mamona, ao passo que, nos grãos, a quantidade de óleo concentra-se em torno de $40 \%$ na semente de girassol, $18 \%$ na soja e $44 \%$ no milho. Dessa forma, as microalgas são oportunas à produtividade do biodiesel, apresentando alto rendimento em óleo, crescimento em curto espaço de tempo, otimizadas em uma temperatura de $20^{\circ} \mathrm{C}$ à $35^{\circ} \mathrm{C}$, oportunizando a não competição e auxiliando na captura e uso de $\mathrm{CO}_{2}$ (Galina et al., 2020). 
O cultivo de microalgas em ambientes naturais como as lagoas abertas, ou artificiais com auxílio de fotobiorreatores (FBR) são constantemente utilizados. Porém, apesar da empregabilidade econômica no uso de lagoas abertas, os FBR são considerados mais favoráveis devido a não exploração do solo, menor gasto de água, controle das variáveis climáticas além de minimizar as probabilidades de contaminação (Carneiro et al., 2018). De acordo com El Shimi e Moustafa (2017) o fornecimento de nutrientes advindos da água são limitados como meio de crescimento de biomassa, e consequentemente poderiam desencadear um aumento nos gastos com a produção, pois exigem essencialmente fontes nutritivas compostas por macro e micronutrientes.

Para que a conversão da biomassa em biodiesel seja economicamente viável, Xu et al., (2019) contextualizam a importância de ampliar sua produtividade e minimizar os custos com os insumos utilizados para preparar o meio de cultivo sintético, pois totalizam um gasto de aproximadamente $35 \%$ do valor total da produção. Vale ressaltar também a importância de promover as vantagens ambientais, pois o cultivo desses organismos não necessita competir por terras agricultáveis, possuem alta capacidade de capturar $\mathrm{CO}_{2}$ minimizando os danos causados pelo efeito estufa na atmosfera e ainda, as microalgas despertam grande potencial por não serem tendenciosas a causar problemas como desmatamento, erosão do solo e poluição da água quando comparadas a outras fontes de matéria prima para produção de biocombustíveis (Shi; Handler; Shonnard, 2019; Shuba; Kifle, 2018).

Atualmente, há a necessidade em substituir os combustíveis fósseis, que são limitados, por outros que são renováveis, assim a geração de novos combustíveis, como por exemplo o biodiesel poderá ser feito em um futuro com a utilização de microalgas, pois segundo Lima, Porto e Freitas (2018), elas podem possuir alta concentração lipídica para produção de biocombustível. O melhoramento das condições de crescimento de microalgas para maximizar a produção de biomassa em termos de densidade celular das algas e concentração lipídica justifica a necessidade de realização deste trabalho, pois melhorando estas condições é possível aumentar o potencial de utilização desta espécie para produção de biocombustíveis (Benedito; Porto; Freitas, 2019). Diante do exposto, o presente estudo objetiva otimizar o cultivo de uma espécie de Scenedesmus sp. que possibilite uma maior produção de teor lipídico.

\section{Metodologia}

A fim de comparar o comportamento dos cultivos em meios distintos e viabilizar a melhor condição de produção para as amostras de biomassa algal, utilizou-se cepas da microalga Scenedesmus sp. proveniente do Laboratório de Química do Instituto Luterano de Ensino Superior (ILES/ULBRA), isoladas e mantida no LABIOGREENC, Biofuels and green chemistry laboratory da Universidade Federal de Uberlândia (UFU).

\subsection{Condições de produção}

Os meios nutricionais sintéticos utilizados para o cultivo foram o Chu (Chu, 1942) adaptado de Silva (2014) e Guillard (1975) modificado sem adição de vitaminas. Preparou-se as soluções respectivas de cada meio, com as concentrações $\left(\mathrm{g} \mathrm{L}^{-1}\right)$ de cada reagente utilizado para cada litro do meio de cultivo.

As condições operacionais realizadas em escala experimental laboratorial, ocorreram em reatores horizontais, do tipo bandeja aberta em acrílico com volume total de $2 \mathrm{~L}$, mas com volume útil de 1 Litro, fechados com plástico filme de PVC com perfurações alternadas para auxiliar na oxigenação e minimizar a evaporação, mantidos em temperatura ambiente de $25^{\circ} \mathrm{C} \pm 1{ }^{\circ} \mathrm{C}$, com controlador de fotoperíodo em 12 horas de luz e 12 horas de escuro, utilizando 3 lâmpadas fluorescentes brancas de 40 Volts (Figura 1), semelhante à unidade experimental adotada por Ramirez, Farenzena, Trierweiler (2014). 
Figura 1 - Condições operacionais para o cultivo de Scenedesmus sp.

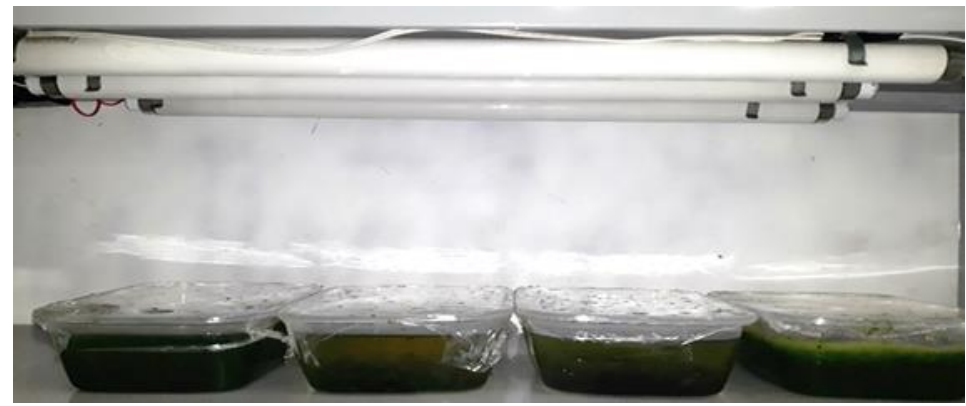

Fonte: Autores (2021).

Para verificar se o tempo de cultivo (dias) e a porcentagem de inóculo causariam interferências na produtividade de microalga Scenedesmus sp., junto ao meio sintético de cultivo, as condições experimentais de produção foram otimizadas com base em um planejamento fatorial $2^{3}$, no qual cada variável (meio de cultivo, tempo e \% de inóculo) foi estudada em dois níveis, realizando um total de 8 experimentos a partir da combinação de todas as condições.

O meio de cultivo, o tempo e a porcentagem de inóculo são variáveis importantes para o crescimento das algas e, portanto, para a quantidade de lipídeos formadas por elas, neste sentido e visando minimizar o número de experimentos realizados e o tempo gasto, utilizou-se o planejamento fatorial $2^{3}$.

A partir das variáveis selecionadas e seus níveis, foi construída a matriz do planejamento fatorial $2^{3}$, que apresenta a combinação de todas as condições experimentais para o cultivo das algas visando a produção de lipídeos. A Tabela 1 apresenta a matriz do planejamento de experimentos, realizada para otimização do processo de cultivo das algas estudadas.

Tabela 1 - Matriz do planejamento experimental fatorial $2^{3}$, para variações no meio de Cultivo. Variáveis: M: Meio de Cultivo, T: Tempo de cultivo em dias e I: Porcentagem de Inóculo.

\begin{tabular}{|c|c|c|c|c|c|c|}
\hline \multirow[b]{2}{*}{ Ensaios } & \multicolumn{6}{|c|}{ Variáveis } \\
\hline & M & $\begin{array}{l}\text { Meio de } \\
\text { cultivo }\end{array}$ & $\mathrm{T}$ & Tempo (dias) & I & \% de inóculo \\
\hline 1 & - & Chu & - & 20 & - & 25 \\
\hline 2 & + & Guillard & - & 20 & - & 25 \\
\hline 3 & - & Chu & + & 30 & - & 25 \\
\hline 4 & + & Guillard & + & 30 & - & 25 \\
\hline 5 & - & Chu & - & 20 & + & 35 \\
\hline 6 & + & Guillard & - & 20 & + & 35 \\
\hline 7 & - & Chu & + & 30 & + & 35 \\
\hline 8 & + & Guillard & + & 30 & + & 35 \\
\hline
\end{tabular}

Fonte: Autores (2021).

Para colheita de biomassa e determinação de sua massa seca $\left(\mathrm{g} \mathrm{L}^{-1}\right)$, os volumes totais dos reatores foram submetidos a centrifugação (AAKER), a $4000 \mathrm{rpm}$ por 5 minutos. O sobrenadante composto pelo meio de cultivo, foi descartado para que o sedimento de biomassa fosse alocado em béquer previamente pesados, levados para secagem em estufa a $80{ }^{\circ} \mathrm{C}$ por 48 horas (Figura 2). Para realizar a quantificação de biomassa seca final, expressas em $\mathrm{g} \mathrm{L}^{-1}$, as amostras foram pesadas de acordo com 
a metodologia indicada por Ramirez, Farenzena e Trierweiler (2014).

Figura 2 - Processo de colheita e secagem de biomassa da microalga Scenedesmus sp.

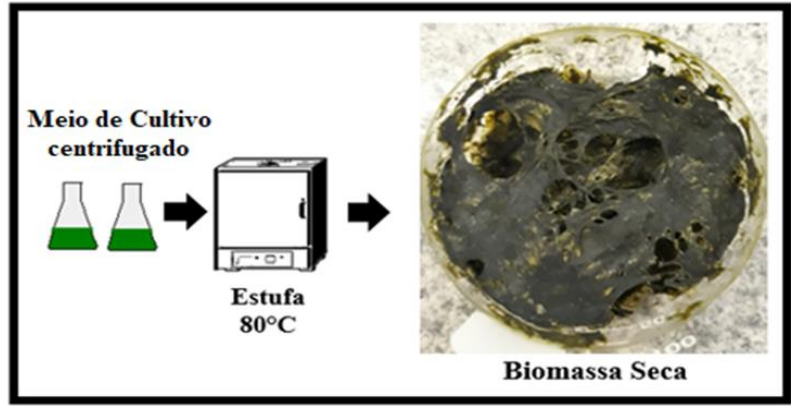

Fonte: Autores (2021).

\subsection{Extração de lipídeos}

Após a colheita de biomassa, seguida de centrifugação e secagem, o total da amostra (aproximadamente $1000 \mathrm{mg}$ ) foi submetida à extração de lipídeos, conforme metodologia descrita por Folch; Lees; Stanley (1957). Inicialmente, a biomassa foi macerada em cadinho de porcelana adicionando $40 \mathrm{~mL}$ de clorofórmio e $20 \mathrm{~mL}$ de metanol. Em seguida, a mistura foi transferida para um erlenmeyer de $125 \mathrm{~mL}$ submetida a banho ultrassom (QUIMIS - Ultrassonic Cleanner 1400, frequência de $40 \mathrm{kHz}$ ) por 90 min para auxiliar na ruptura celular. Logo após, a amostra foi centrifugada, conforme sequência apresentada na Figura 3.

Figura 3 - Extração de óleo da Scenedesmus sp. utilizando metodologia proposta por Folch; Lees; Stanley (1957).

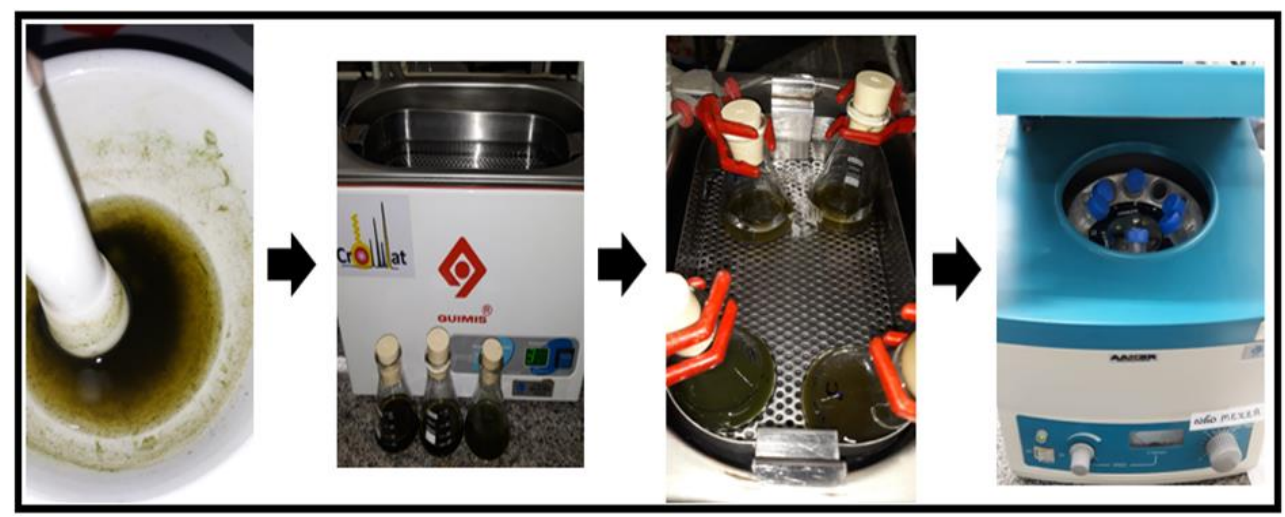

Fonte: Autores (2021).

Ao final dos ciclos, em que a amostra foi centrifugada por $8 \mathrm{~min}$, a parte sólida sedimentou-se e o sobrenadante contendo os lipídeos foi transferido para um béquer contendo $12 \mathrm{~mL}$ de solução $\mathrm{KCl} 0,88 \%$ (m/v). Esse foi submetido a agitação e encaminhados para o funil de separação. Após a formação de duas fases, reservou-se a fase contendo os lipídeos, adicionando $4 \mathrm{~mL}$ de mistura metanol:água 1:1, submetendo o funil com a mistura à nova agitação. A fase superior foi retirada por sucção e descartada, enquanto a fase inferior contendo lipídeos foi filtrada com sulfato de magnésio. Em seguida, o filtrado foi submetido a completa evaporação dos solventes em capela de exaustão, conforme apresentado na Figura 4. 
Figura 4 - Processo representativo do sistema de extração de óleo da microalga Scenedesmus sp. utilizando, funil de separação, secagem com sulfato de magnésio, filtragem e capela de exaustão.

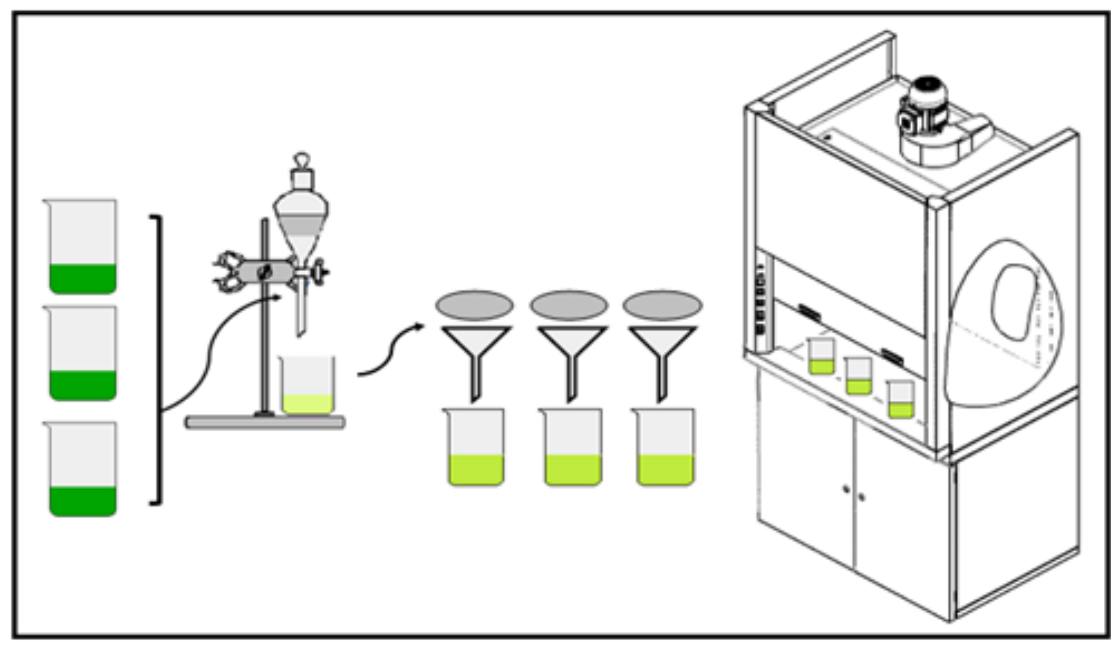

Fonte: Autores (2021).

Posteriormente, a massa dos frascos contendo o óleo produzido em cultivo, foi aferida para que o cálculo gravimétrico fosse realizado obtendo a mensuração do teor lipídico total em porcentagem de acordo com a Equação 1.

Teor de lipídeos $(\%)=($ massa de óleo produzida $\times 100) /($ massa da biomassa $\times 1 \mathrm{~L})$

Eq. 1

Sendo: massa de óleo em gramas e massa da biomassa em gramas por litro.

\subsection{Otimização das condições de cultivo}

A partir do planejamento fatorial $2^{3}$ foi possível verificar quais variáveis empregadas ao meio de cultivo proporcionaram os melhores resultados para produção de biomassa, expressa em $\mathrm{g} \mathrm{L}^{-1}$ e de óleo indicando o teor lipídico em porcentagem mássica. Assim, este planejamento está sendo realizado com a finalidade de selecionar as condições que apresentarem melhor desempenho em relação às variáveis estudadas para serem mais bem avaliados em um planejamento composto central (PCC).

O PCC foi realizado com duas variáveis, resultando em 11 experimentos que combinam os níveis $(+1$ e -1$)$ das variáveis em um planejamento fatorial $2^{2}$, os níveis $(+\alpha$ e $-\alpha)$ das variáveis em um planejamento axial e três repetições no ponto central. As variáveis estudadas foram a concentração de nutrientes do meio Chu (\%) e o tempo de cultivo (dias). A resposta dos experimentos foi o teor de lipídeos, determinado pelo método proposto por Folch, Lees e Stanley (1957). A Tabela 2 apresenta as variáveis e os níveis estudados na otimização da extração de lipídeos da, enquanto a Tabela 3 apresenta a matriz de experimentos do PCC combinando os níveis das variáveis codificadas.

Tabela 2 - Variáveis e níveis da otimização do teor lipídico.

\begin{tabular}{lllllll}
\hline Variável & Nível & & & & \\
& & $\mathbf{- 1 , 4 1}$ & $\mathbf{- 1}$ & $\mathbf{0}$ & $\mathbf{1}$ & $\mathbf{1 , 4}$ \\
\hline ( 1 ) Concentração Meio Chu (\%) & 40 & 50 & 75 & 100 & 110 \\
( 2 ) & Tempo (dias) & 12 & 15 & 22 & 30 & 33 \\
\hline
\end{tabular}

Fonte: Autores (2021). 
Tabela 3 - Matriz de planejamento composto central em 2 variáveis para otimização do teor lipídico (1- Concentração Meio Chu em porcentagem (\%) e 2 - Tempo em dias).

\begin{tabular}{ccccc}
\hline Experimento & \multicolumn{2}{c}{ Codificado } & \multicolumn{2}{c}{ Valores Reais } \\
\cline { 2 - 5 } & $\mathbf{1}$ & $\mathbf{2}$ & Meio & Tempo \\
\hline $\mathbf{1}$ & - & - & 50 & 15 \\
$\mathbf{2}$ & + & - & 100 & 15 \\
$\mathbf{3}$ & - & + & 50 & 30 \\
$\mathbf{4}$ & + & + & 100 & 30 \\
$\mathbf{5}$ & 0 & 0 & 75 & 22 \\
$\mathbf{6}$ & 0 & 0 & 75 & 22 \\
$\mathbf{7}$ & 0 & 0 & 75 & 22 \\
$\mathbf{8}$ & $-1,41$ & 0 & 40 & 22 \\
$\mathbf{9}$ & $+1,41$ & 0 & 110 & 22 \\
$\mathbf{1 0}$ & 0 & $-1,41$ & 75 & 12 \\
$\mathbf{1 1}$ & 0 & $+1,41$ & 75 & 33 \\
\hline
\end{tabular}

Fonte: Autores (2021).

As análises estatísticas foram realizadas com auxílio do Software Statistica 7.1 (StatSoft) explorando a importância e os efeitos das variáveis na eficiência da extração lipídica, utilizando a metodologia de superfície de resposta para determinar as condições ótimas para a porcentagem máxima de extração lipídica.

\section{Resultados e Discussão}

Serão apresentados abaixo os resultados dos experimentos realizados dentro do planejamento fatorial $2^{3}$, para verificar quais das variáveis estudadas são significativas ou não para produção de biomassa da microalga e teor lipídico.

\subsection{Avaliação de crescimento da microalga Scnedesmus sp. em cultivos com meio Chu e Guillard}

Nos ensaios 1 e 3 a microalga Scenedesmus sp. foi cultivada em meio Chu, em período de 20 dias (ensaio 1) e 30 dias (ensaio 3). Com relação aos ensaios 2 e 4 o cultivo da microalga Scenedesmus sp. teve como nutrientes disponíveis o meio Guillard, em período de 20 dias (ensaio 2) e 30 dias (ensaio 4). O crescimento celular de ambos os quatro cultivos foi monitorado a cada cinco dias por absorbância a $570 \mathrm{~nm}$, analisando a densidade óptica. Verifica-se na Figura 5 o perfil de crescimento das algas (Absorbância) nos respectivos tempos de cultivo analisados. 
Figura 5 - Crescimento celular analisado nos ensaios experimentais em meio nutricional Chu e Guillard.

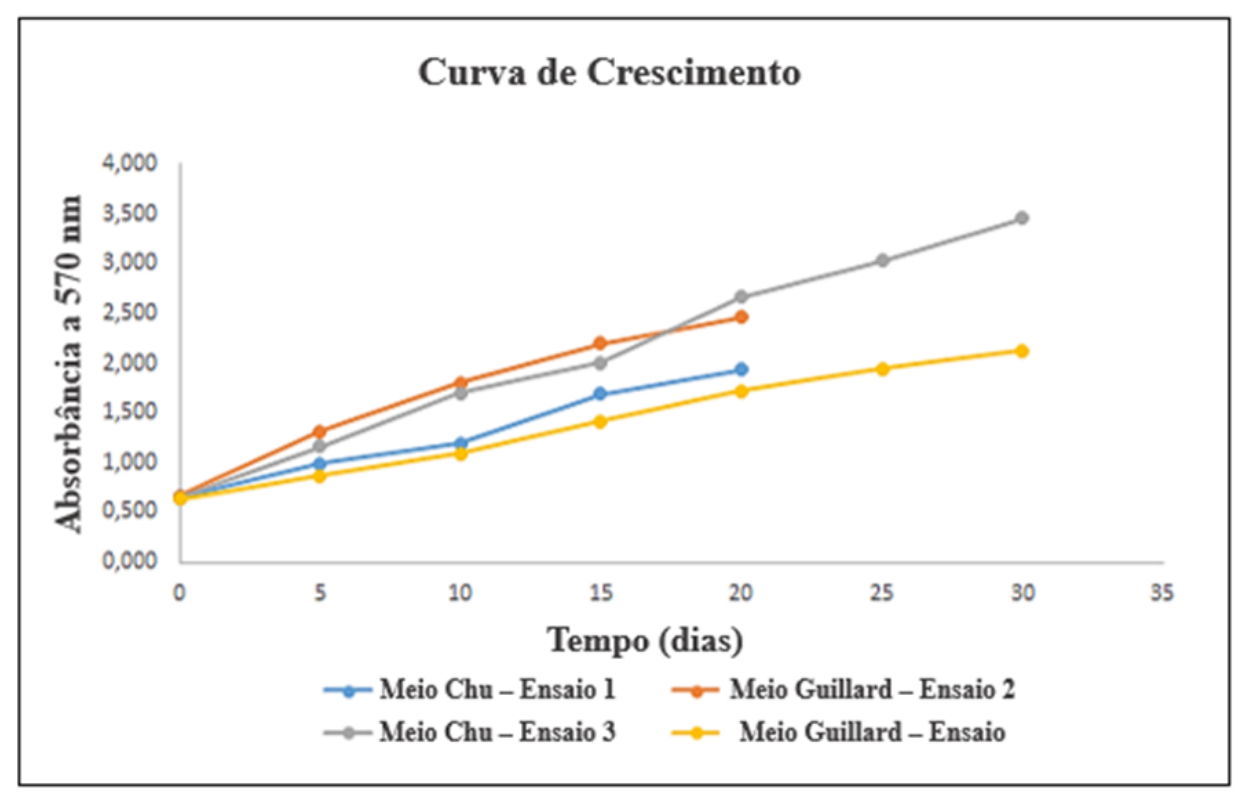

Fonte: Autores (2021).

Observa-se que a densidade celular da microalga originou uma fase de crescimento gradativo em todos os ensaios experimentais. Os resultados do ensaio 2 mantiveram-se superiores ao ensaio 1 cerca de $21 \%$ mais eficaz, ao serem submetidos a um tempo de cultivo de 20 dias. Quando relacionados os ensaios 3 e 4, o percentual de desenvolvimento é ainda mais satisfatório, o ensaio 3 apresentou em seus parâmetros taxa de crescimento elevada na ordem de 53\% a mais que o estimado para o ensaio 4. Ambos foram condicionados ao cultivo de 30 dias.

Esses dados mostram que é possível desenvolver um cultivo durante um período de 30 dias otimizando as taxas de crescimento dos organismos, visto que a evolução das microalgas apresenta desenvolvimento até por volta dos 30 dias de cultivo, quando se inicia um período de decréscimo celular, indicativo do declínio dos nutrientes com o passar do tempo, após consumo dos mesmos pelas microalgas (Dourado et al., 2020).

\subsection{Ensaios realizados para otimização no cultivo de microalgas}

A biomassa, produzida dos meios de cultivo, foi submetida a centrifugação e secagem para extração de óleo conforme metodologia descrita por Folch, Lees e Stanley (1957). Posteriormente, a massa dos frascos contendo óleo produzido foi aferida para que o cálculo gravimétrico fosse realizado obtendo ao final do processo metodológico a mensuração do teor lipídico total em porcentagem. A metodologia de cultivo estudada com intuito de avaliar se as variáveis selecionadas possuem influência na formação lipídica da microalga Scenedesmus sp., foi a partir de um planejamento fatorial $2^{3}$. As três variáveis escolhidas foram: meio de cultivo, tempo de cultivo em dias e porcentagem de inóculo, empregando dois níveis para cada variável. A Tabela 4 apresenta a matriz do planejamento de experimentos e as interações entre as variáveis, a fim de observar a influência de uma variável sobre o rendimento lipídico no cultivo das microalgas. 
Tabela 4 - Matriz do planejamento de experimentos com resultados do teor lipídico obtido das algas de acordo com o planejamento fatorial $2^{3}$ (M: meio de cultivo; T: tempo de incubação; I: \% de inóculo; \% TL: porcentagem do teor lipídico).

\begin{tabular}{lllllllll}
\hline \multirow{2}{*}{ Ensaios } & \multicolumn{2}{l}{ Variáveis } & \multicolumn{1}{c}{} & & & \% TL \\
\cline { 2 - 7 } & $\mathbf{M}$ & $\mathbf{T}$ & $\mathbf{I}$ & $\mathbf{M} \times \mathbf{T}$ & $\mathbf{M} \times \mathbf{I}$ & $\mathbf{T} \times \mathbf{I}$ & $\mathbf{M} \times \mathbf{T} \times \mathbf{I}$ & \\
\hline $\mathbf{1}$ & - & - & - & + & + & + & - & 1,70 \\
$\mathbf{2}$ & + & - & - & - & - & + & + & 0,29 \\
$\mathbf{3}$ & - & + & - & - & + & - & + & 10,87 \\
$\mathbf{4}$ & + & + & - & + & - & - & - & 0,50 \\
$\mathbf{5}$ & - & - & + & + & - & - & + & 5,23 \\
$\mathbf{6}$ & + & - & + & - & + & - & - & 1,94 \\
$\mathbf{7}$ & - & + & + & - & - & + & - & 11,02 \\
$\mathbf{8}$ & + & + & + & + & + & + & + & 3,41 \\
\hline
\end{tabular}

Fonte: Autores (2021).

Com os resultados obtidos do teor de lipídeos calculou-se os efeitos das variáveis em cada ensaio. Os efeitos para cada variável e interação entre as variáveis foram obtidos a partir da diferença entre as respostas médias de teor de lipídeos nos níveis superior e inferior para cada variável, conforme Equação 2.

Efeito (Ef) = Média de \%TL (nível +) - Média de \%TL (nível -)

Eq. 2

Em que $\left(E_{f}\right)$ é o efeito da variável; Média \%TL (nível +) corresponde à porcentagem média do teor lipídico obtido para o nível codificado +1 das variáveis; Média \% TL (nível +) corresponde à porcentagem média do teor lipídico obtido para o nível codificado -1 das variáveis, obtendo os valores de efeito apresentados na Tabela 5.

Tabela 5 - Efeitos das variáveis estudadas e suas interações na porcentagem de lipídeos obtidas no cultivo da microalga.

\begin{tabular}{ccc}
\hline & Variáveis e suas interações & Efeitos \\
\hline 1 & Meio de Cultivo & $-5,66$ \\
2 & Tempo de cultivo (dias) & $+4,17$ \\
3 & Porcentagem de inóculo (\%) & $+2,07$ \\
12 & Meio x tempo & $-3,33$ \\
13 & Meio x inóculo & $+0,21$ \\
23 & Tempo x inóculo & $-0,54$ \\
123 & Meio x tempo x inóculo & $+1,17$ \\
\hline
\end{tabular}

Fonte: Autores (2021).

De acordo com os valores de efeito, a porcentagem de inóculo, dentro dos níveis estudados (25 e $35 \%$ ), apresentou a menor influência no teor de lipídeos produzidos. O tempo de cultivo e o meio apresentaram efeitos próximos, com maior importância para o meio de cultivo usado. O sinal negativo do efeito do meio de cultivo indica que o nível baixo possui maior influência na porcentagem de lipídeos, ou seja, o meio Chu se mostra mais promissor no cultivo das algas para obtenção de lipídeos do que o meio Guillard. 
Como os experimentos não foram realizados em replicatas, o erro dos efeitos não pôde ser estimado a partir de variâncias individuais dos experimentos. Mas existe outra forma de estimar o erro dos efeitos sendo realizada pela análise do gráfico normal dos efeitos, no qual os efeitos mais dispersos de um valor padronizado (Z) igual a zero são estatisticamente significativos. Para construir o gráfico normal de efeitos foi realizado o cálculo das interações entre os fatores, resultando no total de sete efeitos: três efeitos principais, três interações de dois fatores e uma interação de três fatores. Para o cálculo dos efeitos de interação de variáveis multiplicou-se os sinais dos níveis de elemento a elemento. Primeiro, as interações das variáveis são determinadas pela multiplicação dos níveis de duas a duas, depois de três a três variáveis (Barros Neto; Scarmínio; Bruns, 2001). A significância dos efeitos pode ser melhor observada através do gráfico normal dos efeitos, Figura 6.

Figura 6 - Gráfico normal de efeitos para o cultivo das algas. Sendo que 1 corresponde ao meio de cultivo, 2 ao tempo de cultivo e 3 à porcentagem de inóculo.

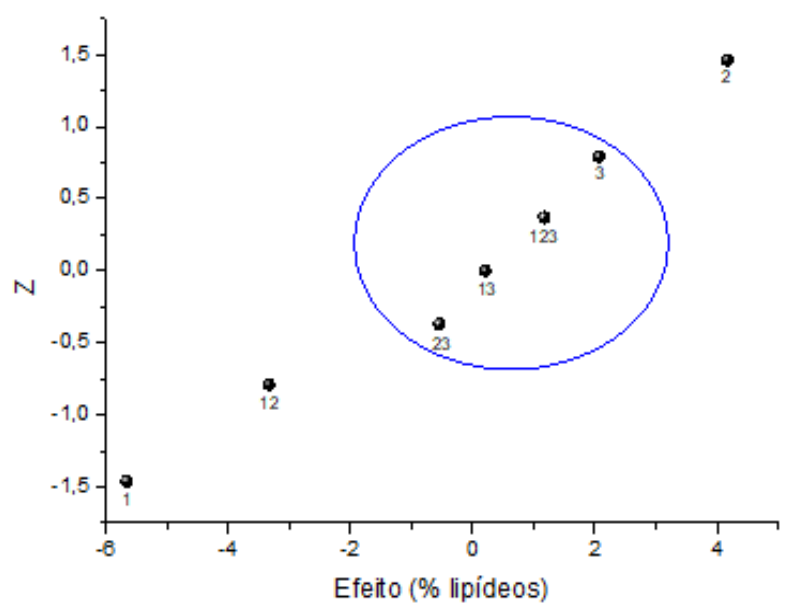

Fonte: Autores (2021).

Os valores de efeitos mais dispersos de um valor padronizado (Z) igual a zero são estatisticamente significativos, e aqueles que se aproximam deste valor não possuem influência significativa na resposta. Na Figura 6 observa-se que os pontos nas extremidades são os mais dispersos e, por isso, são os dois únicos significativos para o cultivo das algas. No círculo azul estão os valores de efeito menos significativos, sendo uma variável discreta, e as demais interações entre as variáveis. A variável em questão, mostrada como o ponto 3 no gráfico, se refere a porcentagem de inóculo e como já havia visto anteriormente, teve pouco influência no teor de lipídeos. A interação indicada pelo ponto 12 está fora da área marcada, porque se distancia de zero, porém como uma interação tem força menor em relevância para a resposta quando comparada a uma variável principal, contudo nos indica que não apenas o meio de cultivo (ponto1) e o tempo (ponto 2) são importantes, coexiste uma resposta positiva à interação destas duas variáveis. O meio de cultivo (ponto 1), está na área negativa do gráfico, portanto precedido pelo sinal negativo que nos indica que o nível baixo (meio $\mathrm{Chu}$ ) foi o que proporcionou melhor resultado para a produção de lipídeo. Com o tempo de cultivo (ponto 2) ocorreu o contrário, ele está na região positiva do gráfico e o nível alto (30 dias) foi mais importante para a produção de lipídeos.

\subsection{Avaliação geral de biomassa seca e teor lipídico produzido}

A Tabela 6 apresenta os resultados obtidos através de um conjunto de variáveis, pelo qual analisou-se o crescimento da microalga Scenedesmus sp. (SCN) em meio Chu e Guillard, adicionando 25 e $35 \%$ de inóculo, a serem cultivados em um prazo entre 20 dias e 30 dias, em ensaios distintos. 
Tabela 6 - Biomassa seca, quantidade de óleo produzido e teor lipídico das microalgas Scenedesmus sp.

\begin{tabular}{cccc}
\hline Ensaios & Biomassa $\left(\mathrm{g} \mathrm{L}^{-1}\right)^{*}$ & Óleo $(\mathrm{g})$ & $\begin{array}{c}\text { Teor lipídico } \\
(\%)\end{array}$ \\
\hline 1 & & & 1,70 \\
2 & 1,32 & 0,022 & 0,29 \\
3 & 1,67 & 0,004 & 10,87 \\
4 & 2,26 & 0,246 & 0,50 \\
5 & 1,08 & 0,005 & 5,23 \\
6 & 1,57 & 0,082 & 1,94 \\
7 & 0,93 & 0,018 & 11,02 \\
8 & 1,76 & 0,194 & 3,41
\end{tabular}

* quantidade produzida por reator com capacidade de 1 litro. Fonte: Autores (2021).

No ensaio 1, aplicou-se Meio Chu acondicionando $25 \%$ do volume útil do reator de inóculo, em um prazo de cultivo de 20 dias, foi encontrado uma produtividade de biomassa final da ordem de 1,3216 $\mathrm{g} \mathrm{L}^{-1}$. Em um experimento conduzido por uma microalga também pertencente à família Scenedesmaceae, cultivada em meio sintético com temperatura de $25 \pm 2{ }^{\circ} \mathrm{C}$ e luminosidade proveniente de lâmpadas fluorescentes de $40 \mathrm{~W}$ com fotoperíodo de $12 \mathrm{~h} / 12 \mathrm{~h}$ de luz/escuro, apresentou crescimento máximo de biomassa equivalente a $1,21 \mathrm{~g} \mathrm{~L}^{-1}$ ao final do $28^{\circ}$ dia de cultivo, obtendo em um maior tempo de cultivo um menor rendimento.

Referente ao teor de lipídeos extraído da biomassa seca, o ensaio 1 apresentou percentual de 1,70\%, considerado baixo por Oliveira et al. (2014) frente a média padrão de 19,6 a 21,1 \% para Scenedesmus sp. No entanto, é importante ressaltar que o ensaio 1 apresentou melhor rentabilidade, 1,48 \% a mais de lipídeos em sua extração quando comparado ao experimento realizado por Erazo (2017), que expõe em sua pesquisa um cultivo com a microalga Scenedesmus sp. em FBR de placa plana durante um período de 15 dias, ressaltando assim a necessidade de se investir em otimizações no processo de cultivo para elevar o rendimento lipídico final.

Mantendo o período de 20 dias de cultivo com adição de inóculo referente a $25 \%$, apresentando variação apenas quanto ao meio quando comparado os parâmetros com o ensaio 1, estabeleceu-se as variáveis do ensaio 2 , o qual utilizou Guillard como meio base, resultando em uma maior quantidade de biomassa seca produzida $\left(1,67 \mathrm{~g} \mathrm{~L}^{-1}\right)$. Desempenho semelhante obtido por Pena et al., (2017), em seu plano experimental estava sob intensidade luminosa, utilizando fotoperíodo de $12 \mathrm{~h}$ luz e $12 \mathrm{~h}$ escuro, à uma temperatura ambiente $\left(25^{\circ} \mathrm{C}\right)$, durante período de 20 dias, ressaltou em seus resultados concentrações máximas equivalentes a $1,75 \mathrm{~g} \mathrm{~L}^{-1}$ para o cultivo da microalga Scenedesmus sp., e 1,64 g L $\mathrm{L}^{-1}$ para a Chlorella sp.

Verificou-se baixa concentração de óleo produzido no ensaio 2 (0,004 g), mensurando um teor lipídico de 0,29\%. Embora ambos tenham proporção de inóculo inicial (25\%) e tempo em cultivo de 20 dias, o ensaio 2 está associado a outro meio sintético, o meio Guillard, determinando uma produção de biomassa de 1,67 $\mathrm{g} \mathrm{L}^{-1}$, maior que valores encontrados por Erazo (2017), que alcançou durante um experimento com a microalga Scenedesmus sp. concentrações de 1,5 g $\mathrm{L}^{-1}$, em ambiente climatizado, iluminado por duas lâmpadas tipo LED tubulares de $12 \mathrm{~W}$ cada uma, em fotoperíodo com $24 \mathrm{~h}$ de claridade. Para o crescimento das microalgas em taxas satisfatórias deve ser observado seu rendimento lipídico, o que indica produção óleo. No ensaio 2 foi utilizado o meio Guillard, o qual desempenhou maior rendimento de biomassa quando comparado ao ensaio 1, porém os dados se diferem frente ao teor de lipídeos produzidos por ambos, sendo que o cultivo submetido ao meio Chu (ensaio 1) indicou 1,3845 \% a mais de óleo. Acredita-se que o processo metabólico possa estar 
condicionado a esse resultado, em que a microalga Scenedesmus sp. estivesse enfrentando dificuldades de se adaptar ao meio de cultivo Guillard ao longo de seu crescimento e multiplicação celular, sendo possível que ela esteja consumindo seu óleo produzido, visto que essa é uma substância de reserva nutricional para continuar o crescimento (Borges et al., 2016). Esse resultado obtido no ensaio 2, onde elevada biomassa indicou baixo teor de óleo comparado as condições atribuídas ao ensaio 1 , foi essencial para constatar que a produção lipídica não está diretamente ligada ao crescimento celular. Há diversas variáveis (temperatura, luz, pH, aeração, nutrientes) que podem interferir no cultivo de microalgas, porém é pertinente mencionar os fatores biológicos que variam entre as espécies e seus armazenamentos metabólicos de reservas nutricionais (Borges et al., 2016; Silva, 2014).

Oliveira et al. (2014) otimizando seu meio de cultivo Chu adicionando porcentagem de efluente de bovinocultura biodigerido, para a microalga Scenedesmus sp., empregou parâmetros de temperatura controlada a $22 \pm 2{ }^{\circ} \mathrm{C}$, luminosidade com fotoperíodo 12 h:12 h e bombas de aeração constante, quantificou uma extração lipídica de 14,91\% para $10 \%$ efluente utilizado como meio nutricional adicional.

Para avaliar o perfil do óleo da microalga Scenedesmus sp., como fonte de nutrientes para o cultivo de microalgas, Dzuman (2013) utilizou meio concentrado Chu, com controle de temperatura de $20 \pm 2{ }^{\circ} \mathrm{C}$, aeração, iluminação fluorescente branca de 40 Volts, $12 \mathrm{~h} \mathrm{luz/12} \mathrm{h}$ escuro, obtendo produtividade de biomassa seca equivalente a $677 \mathrm{mg} \mathrm{L} \mathrm{L}^{-1}$ e $16 \%$ de bioóleo. Seguindo essa linha de raciocínio onde o meio Chu vem se destacando, os ensaios 1 e 3 cultivados nas mesmas condições de meio e variação de inóculo, quando submetido ao cultivo por 30 dias (ensaio 3), o experimento apresentou maior concentração de teor lipídico (10,87\%). Indica-se que o tempo de 30 dias, aparentemente é mais promissor do que a variável do ensaio 1 (20 dias). Segundo estudos apontados por Ramirez, Farenzena e Trierweiler (2014), a microalga Scenedesmus sp., entra e fase exponencial de crescimento a partir do décimo dia de cultivo, onde se faz necessário avaliar o ciclo de operação para que o cultivo em fotobiorreatores obtenha a concentração máxima de biomassa em tempo de cultivo favorável com a presença de nutrientes para uma colheita satisfatória.

De acordo com os resultados obtidos por Vieira et al. (2014), a biomassa seca e o teor de lipídeos dessas microalgas foram melhorados quando cultivadas em meio com adição de nutrientes residuais presentes no efluente; apresentando um ter de lipídeos de 10,42\%, resultado ainda inferior ao desempenho obtido pelo cultivo em ensaio 3.

É possível observar que o meio de cultivo Chu vem sempre apresentando melhores resultados, inclusive quando comparados os ensaios 1 e 2, onde ambos apresentavam em seus parâmetros, o mesmo tempo de cultivo (30 dias) e presença de inóculo inicial (25\%), variando apenas o meio. O ensaio 2 cultivado em meio Guillard demonstrou baixo teor lipídico quando comparado ao percentual obtido no ensaio 1 , com cerca de rendimento $83 \%$ menor.

Embora o fator tempo, tenha sido promissor ao rendimento de biomassa quando analisado os ensaios 1 e 3 cultivados em meio Chu, ao serem submetidos a variação de meio, as estirpes que apresentaram crescimento exponencial em meio Guillard, foram cultivadas em 20 dias. Borges et al. (2016), relata em seus resultados, que a microalga Scenedesmus sp., cultivada no meio Guillard em unidade experimental com controle de variáveis de iluminação, temperatura e aeração, entra em estabilidade de crescimento entre o décimo e décimo sexto dia, passando para fase de declínio e morte celular.

A variação em porcentagem de inóculo inicial (35\%) no meio Chu, também foi analisada. No ensaio 5, a biomassa seca quantificada foi superior quando comparada com a biomassa do cultivo desenvolvido nos ensaios 1 , em que ambos estavam em meio Chu, e o mesmo comportamento pode ser observado para o teor lipídeos. Esses resultados sugerem a possibilidade de aumentar a porcentagem de inóculo inicial para $35 \%$, aumentando assim de $68 \%$ a produtividade de teor lipídico e de $16 \%$ a produtividade em biomassa. A concentração de teor lipídico produzida durante o cultivo do ensaio 8 variou significativamente comparado ao ensaio 6, as condições em que as microalgas são submetidas ao meio de cultivo podem afetar seu rendimento metabólico e, assim, consequentemente o acúmulo de lipídeos na célula. Visto que o ensaio 8 
apesar de manter os parâmetros de meio e porcentagem de inóculo, ao alterar o tempo de cultivo os resultados indicaram um aumento de $43 \%$ no teor lipídico.

Na Tabela 6 pode se observar uma relação de otimização nos estudos direcionados ao cultivo da microalga Scenedesmus sp., e seus respectivos rendimentos de teor lipídico. De acordo com os valores observados no ensaio 3 e 7 , percebe-se que os rendimentos variam, dependendo da porcentagem de inóculo. Nota-se que Gouveia et al., (2016) ao cultivar a Scenedesmus em fotobiorreatores com luz contínua e temperatura de $25^{\circ} \mathrm{C}$ obteve teor lipídico de 8,1\%, em um período de 10 dias. Levando-se em conta que no ensaio 7 o rendimento em teor lipídico foi de 11,02 \%, a alteração na porcentagem de inóculo não apresentou dados significativos, ficando sugestivo a aplicação de novas variáveis frente ao tempo de cultivo e ao meio Chu, para tentar obter um rendimento maior de teor lipídico, à medida que os parâmetros relacionados ao meio Chu e ao tempo se destacaram entre as variáveis.

\subsection{Otimização das condições de cultivo}

Para otimização do teor lipídico extraído das microalgas foram realizados experimentos embasados em um planejamento estatístico do tipo planejamento composto central (PCC), analisando as variáveis, concentração de nutrientes do meio Chu (\%) e o tempo de cultivo (dias), conforme disposto na Tabela 7. Como resposta dos experimentos, a partir desse modelo estatístico, foram obtidas como respostas o teor de lipídeos, expresso em \%, a quantidade de óleo produzidos, em gramas e biomassa seca $\left(\mathrm{g} \mathrm{L}^{-1}\right)$. Todos os ensaios experimentais foram realizados em duplicatas, e os resultados apresentados são a média dos valores obtidos.

Tabela 7 - Biomassa seca, quantidade de óleo produzido e teor lipídico das microalgas Scenedesmus sp. em processo de otimização de cultivo.

\begin{tabular}{cccc}
\hline Ensaios & Biomassa $\left(\mathbf{g ~ L}^{\mathbf{- 1}}\right)^{*}$ & Óleo $(\mathbf{g})$ & Teor lipídico (\%) \\
\hline $\mathbf{1}$ & 1,0255 & 0,0258 & 2,5 \\
$\mathbf{2}$ & 0,9992 & 0,0243 & 2,6 \\
$\mathbf{3}$ & 0,9360 & 0,0092 & 9,9 \\
$\mathbf{4}$ & 0,9682 & 0,0124 & 1,2 \\
$\mathbf{5}$ & 0,8730 & 0,0738 & 8,4 \\
$\mathbf{6}$ & 1,0602 & 0,0956 & 9,0 \\
$\mathbf{7}$ & 1,1157 & 0,0963 & 8,6 \\
$\mathbf{8}$ & 0,8957 & 0,1336 & 14,9 \\
$\mathbf{9}$ & 0,9870 & 0,0347 & 1,5 \\
$\mathbf{1 0}$ & 0,9797 & 0,0394 & 4,0 \\
$\mathbf{1 1}$ & 1,0887 & 0,0689 & 6,3 \\
\hline
\end{tabular}

* quantidade produzida por reator com capacidade de 1 litro. Fonte: Autores (2021).

O modelo matemático obtido a partir do planejamento composto central com 2 níveis gerou uma superfície de resposta e curva de contorno, que são apresentados na Figura 7. A superfície de respostas possibilitou observar que a máxima porcentagem de lipídeos alcançada ocorre quando se tem uma menor concentração de meio Chu em um tempo estimado de 22 dias, dentro das condições experimentais. 
Figura 7 - Superfície de resposta sendo influenciada pela variação de porcentagem nutricional em Meio Chu e tempo em dias.

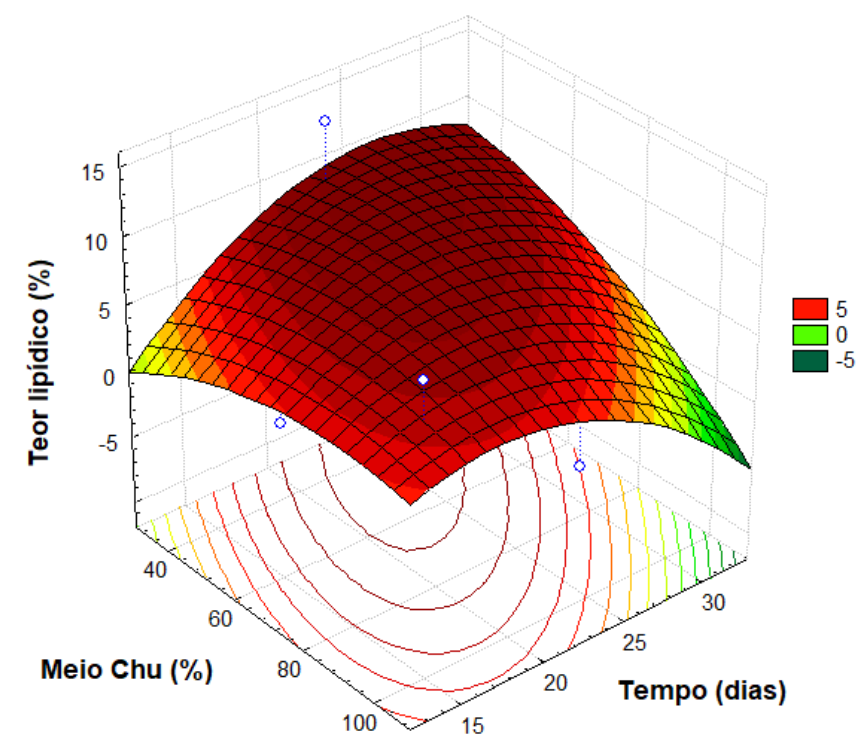

Fonte: Autores (2021).

Na Figura 7, é apresentada a superfície de resposta que descreve o comportamento do acúmulo de lipídeos frente à influência das variáveis, concentração de meio Chu e tempo de cultivo em dias. Foi verificado que a concentração de nutrientes apresentou um eixo sobre a resposta, pois o acúmulo de lipídeos atinge um valor máximo quando a concentração inicial de meio Chu é de aproximadamente 39,5\%. Verificou-se ainda que para valores inferiores ou superiores a este, o modelo prevê uma redução no acúmulo de lipídeos. O tempo de cultivo apresentou um ponto de máximo, indicando a tendência de maior acúmulo de lipídeos em 20 dias de cultivo. Para essas condições de concentração inicial de meio Chu (39,5\%) e tempo de cultivo (20 dias), o modelo prevê um acúmulo de teor lipídico de aproximadamente 9,74\%.

Ao longo da fase de crescimento constante e multiplicação celular o processo nutritivo das microalgas reflete diretamente na constituição bioquímica, assim, porcentagens inferiores do meio nutricional disponíveis, possibilitam taxas mais elevadas de teor lipídico, devido a perda da multiplicação celular a qual concede o lugar ao acúmulo energético em forma de lipídeos (Trevisan; Branco; Arroyo, 2019). Logo, os ensaios que apresentaram concentrações inferiores de nutrientes disponíveis ao meio de cultivo, proporcionam maiores resultados no teor de lipídeos totais, devido ao decréscimo da multiplicação celular que possibilita o acúmulo de energia na forma de lipídeos, conforme mencionado por Trevisan, Branco e Arroyo (2019), o qual avalia a influência da concentração dos nutrientes, nitrogênio e fósforo, em suas condições experimentais, obtendo um rendimento de $12,7 \%$ à 17,9 \% de teor lipídico, demonstrando assim que a porcentagem lipídica é elevada, a medida que ocorre restrição nutricional.

Considerando que o custo com o meio nutricional de cultivo para microalgas é dispendioso, sendo um dos fatores limitantes sua produção e conversão em biodiesel, Monção (2018) realizou um experimento variando a concentração de meio nutricional, com o objetivo de obter uma relação ótima entre a diluição do meio e a produtividade da microalga, onde optou por seguir a metodologia de extração adaptada do método de Folch, Lees e Stanley (1957), obtendo 8,3 \% de lipídeos totais. No que se refere a produção de biodiesel por meio da utilização de biomassa microalgal é indispensável que o cultivo apresente um alto rendimento lipídico. Assim, Guarieiro (2019) desenvolveu uma metodologia com variação na concentração de nutrientes adicionando um efluente oriundo da produção de óleo de palma, com duração de 21 dias, alcançando uma produção $11,21 \% \operatorname{com} 2,12 \mathrm{~g} \mathrm{~L}^{-1}$ de conteúdo lipídico. Ao variar a concentração do meio nutricional, com base em adição de um efluente oriundo de vinhaça, Guarieiro (2019) não registrou um teor de lipídeos totais expressivo (5,74 \%), sendo 
significativamente inferior ao obtido no presente estudo de $9,74 \%$.

Microalgas associadas a três classes distintas (Chlorophyceae, Bacillariophyceae e Cyanophyceae), cultivadas em meio Chu padrão com adição de nitrogênio satisfatório para seu crescimento, demonstrou rendimentos de lipídeos totais variados (na faixa de 3 a $32 \%$ ), devendo-se relacionar os fatores físico-químicos e nutricionais frente ao comportamento metabólico (Fenerick D; Fenerick L, 2020).

A porcentagem lipídica extraída dos experimentos em questão, se mostrou inferior (de 1,2 \% a 14,9 \%), quando correlacionada com a literatura, onde a microalga Scenedesmus, por exemplo apresenta de 19,6 a 21,1\% de lipídeos (Oliveira et al., 2014). Sendo necessário maior otimização das condições de cultivo empregadas, acredita-se que a ausência de aeração tenha sido um fator limitante, podendo evitar a sedimentação celular, mantendo o meio homogêneo, contribuindo para um rendimento mais promissor (Borges et al., 2016).

\section{Conclusão}

Os processos biológicos a partir do cultivo de microalgas para produção de biodiesel, apresentam vantagens por serem competentes fixadoras de carbono atmosférico, controlando e prevenindo a poluição; no entanto, para uma produção competitiva é relevante baixar os custos com o meio de cultivo assim como otimizar os processos de colheita e extração lipídica. Neste trabalho, variáveis foram avaliadas para estimar a melhor combinação metodológica, entre tempo de cultivo, porcentagem inicial de inóculo e meio nutricional.

Entre os resultados, de forma geral todos os experimentos apresentaram melhores rendimentos no teor lipídico, quando submetidos ao cultivo em meio Chu. No entanto, observa-se que o acúmulo de lipídeos atinge um valor máximo quando a concentração inicial de meio Chu é de aproximadamente 39,5\%. Verificou-se ainda que a quantidade de dias (tempo) apresentou um ponto de máximo, indicando a tendência de maior acúmulo de lipídeos em 20 dias de cultivo. Para essas condições de concentração inicial de meio Chu (39,5\%) e tempo de cultivo (20 dias), o modelo prevê um acúmulo de teor lipídico de aproximadamente $9,74 \%$. Sugere-se que sejam realizados estudos que possibilitem baixar o custo dos meios de cultivo, assim como de todos os processos envolvidos em sua produção, sendo algo de fundamental relevância para sua implementação.

O processo adaptativo de microalgas, assim como o estudo das melhores interações entre as variáveis analisadas tem grande importância tecnológica, se fazendo necessário o desenvolvimento de projetos que busquem essa otimização direcionada ao melhoramento e aprimoramento das tecnologias existentes e desenvolvimento de novas técnicas que viabilize sua produção em larga escala, aumentando o percentual lipídico através de metodologias que altere os padrões de cultivo, além de outras frentes de trabalhos voltadas ao beneficiamento do biodiesel produzido a partir de microalgas.

\section{Agradecimentos}

Os autores agradecem o apoio financeiro do Conselho Nacional de Desenvolvimento Científico e Tecnológico (CNPq) e à Coordenação de Aperfeiçoamento de Pessoal de Nível Superior (CAPES) - código de financiamento 001.

\section{Referências}

Barros Neto, B.; Scarminio, I. S. \& Bruns, R. E. (2010). Como Fazer Experimentos: Pesquisa e Desenvolvimento na Ciência e na Indústria. Bookman.

Benedito, V. M.; Porto, P. S. S. \& Freitas, R. R. (2019). Modelagem do crescimento de microalgas: Um estudo bibliométrico. Research, Society and Development, 8 (1), e681511. https://doi.org/10.33448/rsd-v8i1.511 
Borges, W. S.; Araújo, B. S. A.; Moura, L. G.; Coutinho Filho, U.; Resende, M. M. D. \& Cardoso, V. L. (2016). Bio-oil production and removal of organic load by microalga Scenedesmus sp. using culture medium contaminated with different sugars, cheese whey and whey permeate. Journal of Environmental Management, 173, 134-140. https://doi.org/10.1016/j.jenvman.2015.11.015

Carneiro, G. A.; Silva, J. J. R.; Oliveira, G. A. \& Pio, F. P. B. (2018). Uso de Microalgas para Produção de Biodiesel. Research, Society and Development, 7 (5), e1075181. https://doi.org/10.17648/rsd-v7i5.250

Cavalcante Filho, P. G.; Buiaianin, A. M. \& Benatti, G. S. S. (2019). A cadeia produtiva agroindustrial do biodiesel no Brasil: um estudo sobre sua estrutura e caracterização. Desenvolvimento Regional em debate, 9, 772-799. https://doi.org/10.24302/drd.v9i0.2252

CHU, S. P. (1942). The influence of the mineral composition if the medium on the growth of planktonic algae. Journal of Ecology, 30 (2), 284-325. https://doi.org/10.2307/2256574

Dourado, M. S.; Cardoso, C. C. A.; Calado, C. S. C.; Frety, R. T. F. \& Sales, E. A. (2020). Microalgas como matéria prima para a produção de compostos lipídicos precursores de combustíveis verdes. Brazilian Journal of Development, 6 (3), 13985-13994. https://doi.org/10.34117/bjdv6n3-316

Dzuman, M. J. (2013). Efeito da reciclagem repetida do meio de cultivo da microalga Scenedesmus sp para a produção de biodiesel. Dissertação de Mestrado, Universidade Federal do Paraná, Curitiba, PR, Brasil. http://hdl.handle.net/1884/36784

El Shimi, H. I. \& Moustafa, S. S. (2017). Biodiesel production from microalgae grown on domestic wastewater: Feasibility and Egyptian case study. Renewable and Sustainable Energy Reviews, 82 (3), 4238-4244. https://doi.org/10.1016/j.rser.2017.05.073

Erazo, R. G. T. P. (2017). Isolamento, seleção e cultivo em meio sintético e vinhaça de microalgas com potencial para a produção de biodiesel. Dissertação de Mestrado, Universidade Estadual Paulista Júlio de Mesquita Filho, Ilha Solteira, SP, Brasil. https://repositorio.unesp.br/handle/11449/151734

Fenerick, D. C. \& Fenerick, L. H. S. (2020). Avaliação do crescimento da microalga Messastrum gracile em meios de cultura comercial e alternativo utilizando melaço de cana-de-açúcar em menor comprimento de luz. Ciência \& Tecnologia, 12 (1), 96-10.

Folch, J.; Lees, M. \& Stanley, G. H. S. (1957). A simple method for the isolation and purification of total lipides from animal tissues. Journal of Biological Chemistry, 226 (1), 497-509. https://doi.org/10.1016/S0021-9258(18)64849-5

Galina, D.; Benedito, V. M.; Freitas, R. R. \& Porto, P. S. S. (2020). Análise da influência da temperatura e do tempo na transesterificação direta da Nannochloropsis Oculata para produção de biodiesel. Research, Society and Development, 9 (7), e655974648. https://doi.org/10.33448/rsd-v9i7.4648

Gouveia, L.; Graça, S.; Sousa, C.; Ambrosano, L.; Ribeiro, B.; Botrel, E. P.; Castro Neto, P.; Ferreira, A. F. \& Silva, C. M. (2016). Microalgae biomass production using wastewater: Treatment and costs. Scale-up considerations. Algal Research, 16, 167-176. https://doi.org/10.1016/j.algal.2016.03.010

Guarieiro, M. S. (2019). Utilização da vinhaça no cultivo da microalga Klebsormidium flaccidum (Kützing) visando à produção de biomassa aplicada ao mercado de biodiesel. Dissertação de Mestrado, Universidade Federal do Espírito Santo, Vitória, ES, Brasil. http://repositorio.ufes.br/handle/10/11115

Guillard R. R. L. (1975). Culture of Phytoplankton for Feeding Marine Invertebrates. In: Smith W.L., Chanley M.H. (eds) Culture of Marine Invertebrate Animals. Springer. https://doi.org/10.1007/978-1-4615-8714-9_3

Lima, K. F. F.; Porto, P. S. S. \& Freitas, R. R. (2018). Métodos de extração de bio-óleo a partir da microalga Nannochloropsis oculata: uma análise bibliométrica. Research, Society and Development, 7 (6), e976190. https://doi.org/10.17648/rsd-v7i6.259

Monção, F. S. (2018). Microalgas e biocombustíveis: integração de cadeias produtivas. Revista Virtual de Química, 10 (4), $999-1017$.

Oliveira, C. J.; Schaffner, R. A.; Cremonez, P. A.; Feroldi, M. \& Teleken, J. G. (2014). Produção de Biodiesel a partir das algas: uma revisão. Journal of Agronomic Sciences, 3, 202-221.

Pena, A. C. C.; Fontoura, J. T.; Trierweiler, L. F. \& Gutterres, M. (2017). Estudo de um consórcio de microalgas na remoção de nutrientes de efluentes de curtume. Revista Eletrônica Científica da UFRGS, 3 (4), 743-752. https://doi.org/10.21674/2448-0479.34.743-752

Ramirez, N. N. V.; Farenzena, M. \& Trierweiler, J. O. (2014). Growth of microalgae Scenedesmus sp. in ethanol vinasse. Brazilian Archives of Biology and Technology, 57 (5), 630-635. https://doi.org/10.1590/S1516-8913201401791

Ramos, L. (2017). Biodiesel: matérias-primas, tecnologias de produção e propriedades combustíveis. Revista virtual de química, 9 (1), $317-369$.

Silva, D. A. (2014). Produção de biomassa de microalgas cultivadas em esgoto sanitário biodigerido visando a produção de biodiesel. Dissertação de Mestrado, Universidade Federal do Paraná, Curitiba, PR, Brasil. https://acervodigital.ufpr.br/handle/1884/35118

Shi, R.; Handler, R. M. \& Shonnard, D. R. (2019). Life cycle assessment of novel technologies for algae harvesting and oil extraction in the renewable diesel pathway. Algal Research, 37, 248-259. https://doi.org/10.1016/j.algal.2018.12.005

Shuba, E. S. \& Kifle, D. (2018). Microalgae to biofuels: 'Promising' alternative and renewable energy, review. Renewable and Sustainable Energy Reviews, 81, 743-755. https://doi.org/10.1016/j.rser.2017.08.042

Svenning, J. B.; Dalheim, L.; Eilertsen, H. C. \& Vasskog, T. (2019). Temperature dependent growth rate, lipid content and fatty acid composition of the marine cold-water diatom Porosira glacialis. Algal Research, 37, 11-16. https://doi.org/10.1016/j.algal.2018.10.009

Trevisan, E.; Branco, K. B. Z. F. \& Arroyo, P. A. (2019). Avaliação termogravimétrica da biomassa de Chlorella vulgaris cultivada em diferentes condições nutricionais. Engevista, 21 (2), 242-255. 
Research, Society and Development, v. 11, n. 1, e9211124494, 2022

(CC BY 4.0) | ISSN 2525-3409 | DOI: http://dx.doi.org/10.33448/rsd-v11i1.24494

Vieira, T. Q.; Ferreira, W. B.; Araújo, H. W. C.; Cunha, T. H. C. S.; Vidal, I. C. A. \& Melo, D. J. N. (2014). Estudo da viabilidade do uso de resíduos no cultivo da microalga Chlorella sp. visando a produção de biocombustíveis. Monografias Ambientais, 13 (4), $3477-3490$. https://doi.org/10.5902/2236130813544

Xu, H.; Lee, U.; Coleman, A. M.; Wigmosta, M. S. \& Wang, M. (2019). Assessment of algal biofuel resource potential in the United States with consideration of regional water stress. Algal Research, 37, 30-39. https://doi.org/10.1016/j.algal.2018.11.002 